\title{
High-flow oxygen therapy v. standard care in infants with viral bronchiolitis
}

\author{
S Murphy, MB BCh, MSc (Med) (Bioethics), DCH (SA) FCPaed (SA), Crit Care (SA); OrcID 0000-0002-7681-6992; \\ E Bruckmann, MB ChB, MMed (Paed), DCH (SA), FCPaed (SA), Crit Care (SA), OrcID 0000-0002-2824-6850; \\ L G Doedens, BSc, MB BCh, FCPaed (SA), Crit Care (SA); \\ A B Khan, MB BCh, MMed (Paed), FCPaed (SA), Crit Care (SA); OrcID 0000-0002-3105-3522; \\ A Salloo, MB BCh, FCPaed (SA), Crit Care (SA); OrcID 0000-0003-2170-8422; \\ S Omar, FCPath (Chem), Crit Care (SA), DCH (SA); OrcID 0000-0001-8494-1518 \\ Intensive Care Unit, Chris Hani Baragwanath Academic Hospital, University of the Witwatersrand, Johannesburg, South Africa
}

Corresponding author: S Murphy (Susan.Murphy@wits.ac.za)

\begin{abstract}
Background. High-flow humidified oxygen (HFHO) therapy has demonstrated benefit in infants with bronchiolitis.
Objectives. To investigate the efficacy of HFHO in infants with moderate to severe viral bronchiolitis, when used outside the paediatric intensive care unit (PICU), in a hospital with limited PICU resources.

Methods. A randomised controlled trial, which enrolled 28 infants between 1 month and 2 years of age, with a clinical diagnosis of acute viral bronchiolitis and moderate to severe respiratory distress. Participants were randomised to receive HFHO $2 \mathrm{~L} / \mathrm{kg} / \mathrm{min}$ or oxygen by nasal cannula/ face mask. Respiratory rate, heart rate, oxygen saturations, and modified TAL (M-TAL) score were measured at baseline, 60 - 90 minutes after starting therapy and at 6- and 12-hourly intervals. The primary outcome evaluated was the improvement in respiratory distress (M-TAL score). The secondary outcome assessed was the need for intubation and ventilation.

Results. There was a significant improvement in respiratory distress (M-TAL score), in infants who received HFHO therapy. Additionally, there was also a reduction in heart rate in the HFHO group as well as a trend to lower intubation rates.

Conclusion. HFHO is a beneficial therapy for infants with moderate-severe viral bronchiolitis. It can be safely used outside the PICU and could potentially reduce the need for intubation and admission to PICU in resource-limited settings.

Keywords. high-flow oxygen; bronchiolitis; paediatric intensive care.
\end{abstract}

South Afr J Crit Care 2020;36(2):109-113. https://doi.org/10.7196/SAJCC.2020.v36i2.438

Contribution of the study. High-flow humidified oxygen (HFHO) is effective in infants with moderate to severe bronchiolitis, and not only in those with milder forms of the disease. It can be safely used outside the paediatric intensive care unit, where adequate respiratory monitoring is available. This is important in low-resource areas where there may be insufficient critical care resources to manage these patients.

High-flow humidified oxygen (HFHO) is a respiratory therapy which allows the administration of an oxygen/air admixture via a nasal cannula at flows greater than $2 \mathrm{~L} / \mathrm{min} \cdot{ }^{[1]}$ The precise amount of oxygen delivered can be independently titrated to the oxygen flow with delivery of up to $100 \%$ oxygen attainable. In addition, the oxygen/air admixture is heated to a temperature of $34{ }^{\circ} \mathrm{C}$ and humidified to 'optimal humidity' with a water content of $44 \mathrm{mg} / \mathrm{L}$.

The benefits of HFHO therapy on the respiratory system appear to be numerous. ${ }^{[2,3]}$ The high inspiratory flows result in reduced work of breathing, as well as washout of nasopharyngeal dead space. By warming and humidifying inspired gas, it firstly reduces the metabolic work of the patient, and secondly, it minimises the pulmonary broncho-constrictor response which is mediated by nasal muscarinic receptors. ${ }^{[4]}$ In this way, both conductance as well as compliance in the lungs is improved. ${ }^{[5]}$ Additionally, flows above $2 \mathrm{~L} / \mathrm{kg} / \mathrm{min}$ appear to provide some positive end-expiratory pressure (PEEP), estimated to be $\sim 4 \mathrm{cmH}_{2} \mathrm{O}$. The amount of PEEP generated appears to be related to both the flow rate and size of the nasal cannula used. ${ }^{[6,7]}$

At the time of embarking on our study, the use of HFHO therapy in infants with a diagnosis of bronchiolitis appeared to be a promising therapy, but its place in the routine management of these infants was not clear in the absence of data from high-quality RCT's. One RCT in infants with moderate bronchiolitis had shown that among infants given $\mathrm{HFHO}$ at $1 \mathrm{~L} / \mathrm{kg} / \mathrm{min}$ compared with those given $2 \mathrm{~L}$ nasal cannula oxygen, there was no difference between groups in terms of the time spent requiring oxygen. ${ }^{[8]}$ This research aimed to test the hypothesis that there would be no difference in respiratory distress (as measured by the Modified TAL (M-TAL) score) in infants with bronchiolitis who have more severe disease (M-TAL score $>6$ and hypoxaemia in room air), when comparing standard oxygen therapy to HFHO therapy.

The primary outcome assessed was the severity of respiratory distress (measured by the M-TAL score), and the secondary outcome 
was the need for intubation and mechanical ventilation in the control (standard oxygen therapy) and intervention (HFHO therapy) groups.

\section{Methods}

This study was conducted in the high-care area of the paediatric admission ward of Chris Hani Baragwanath Academic Hospital. The high-care area is able to accommodate 14 patients. It has the capacity to ventilate 4 patients while they await a bed in the paediatric intensive care unit (PICU). All patients placed on mechanical ventilators are required to be transferred to a PICU within 24 hours of being intubated, according to unit policy. There are, on average, around 8000 paediatric admissions annually to this hospital with only 9 paediatric intensive care unit beds (PICU) available. Unpublished data from this hospital show that of all those patients who are intubated in the high-care unit, and who are assessed by the triage intensivists as being good candidates for ICU, only $63.3 \%$ manage to be admitted to the hospital's PICU. The remaining $36.7 \%$ are unable to be accommodated due to unavailability of resources, despite being assessed as good candidates. This means that the demand for critical care services almost always exceeds availability, resulting in the high-care area within the paediatric admission ward needing to look after patients who should ideally be admitted to a PICU.

Ethics approval was obtained from the Human Research Ethics Committee of the University of the Witwatersrand (ref. no. M170234). First patients were recruited in August 2017.

The study was designed as a prospective randomised control trial intended to recruit 130 participants over a 2-year period. Sample size estimations were based on the key research questions:

1. Continuous repeated measures outcomes (respiratory rate (RR), heart rate (HR), oxygen requirements, oxygen saturation, M-TAL score). For this we required a repeated measures Analysis of Variance (ANOVA) for each outcome. Based on a medium effect size ( $f+0.25), 5$ measurements per group and a correlation between measurements of 0.5 , a sample size of 78 was required.

2. The proportion of patients requiring intubation and ventilation. For this we required a $z$-test for proportions. Based on an estimated proportion of patients requiring intubation and ventilation of $30 \%$ in the standard care group, and $10 \%$ in the HFHO group, a sample size of 138 was required. Assuming a ventilation rate in the HFHO group, a sample size of 78 was required.

\section{Inclusion criteria}

Patients were eligible for inclusion if they had a clinical diagnosis of bronchiolitis and were between the ages of 1 month (or 44 weeks corrected gestational age) and 2 years. Additionally, they needed to be deemed ill enough by the treating clinician to be admitted to the high-care area of the admission ward for observation and to have moderate/severe respiratory distress, as indicated by an M-TAL score $>6$ and significant hypoxaemia (oxygen saturation $<92 \%$ in room air). The M-TAL score was used as a surrogate indicator of the severity of respiratory distress.

\section{Exclusion criteria}

Patients were excluded if they required intubation and ventilation or if there were signs of impending respiratory failure, namely: apnoea, altered mental state, poor perfusion, $\mathrm{PaCO}_{2}>55 \mathrm{mmHg}$ with a $\mathrm{pH}$ $<7.25$ on arterial blood gas. In addition, patients were excluded if they had any of the following conditions: congenital cardiac disease, multi-organ failure/multisystem disease, if they had been previously ventilated, craniofacial abnormalities, nasal pathology or previous surgery/trauma to nasopharynx, decreased level of consciousness, upper airway obstruction/pathology, and all premature infants who were born at a gestational age less than 34 weeks.

\section{Procedure}

Screening of admissions to high care took place daily on weekdays between $08 \mathrm{~h} 00$ and $17 \mathrm{~h} 00$, with eligible participants invited to take part in the research study. Informed consent was obtained from the parents of all infants enrolled. Participants were randomised to either 'standard therapy' or 'high-flow therapy' using pre-filled envelopes. Those who received standard therapy continued treatment as prescribed by their attending doctors including oxygen administration either by nasal cannula or face mask. This information was recorded on the data collection record. Those who were randomised to HFHO were placed on the high-flow nasal cannula interface as per the high-flow oxygen protocol. All other aspects of participants care were left up to the treating physicians.

The procedure for administering HFHO included the following: the nose and mouth of each infant was suctioned, and the patency of their nostrils was ensured before placing the nasal cannula. Each nasal cannula was sized according to the manufacturer recommendations, ensuring at least $2 \mathrm{~mm}$ between the nasal septum and the prongs. The flow rate was set at $2 \mathrm{~L} / \mathrm{kg} / \mathrm{min}$ for infants up to $10 \mathrm{~kg}$ in weight and for those above $10 \mathrm{~kg}$, an additional $0.5 \mathrm{~L} / \mathrm{kg}$ for each $1 \mathrm{~kg}$ above $10 \mathrm{~kg}$. The inspired oxygen was set at 0.6 at baseline and did not exceed this during the study period. Monitors to measure heart rate and oxygen saturations were continuously applied. Standard oxygen therapy was administered either by nasal cannula $(2 \mathrm{~mm}$ diameter) at $2 \mathrm{~L} / \mathrm{min}$ or Venturi $40 \%$ facemask at $8 \mathrm{~L} / \mathrm{min} ; 100 \%$ oxygen was administered via an oxygen flowmeter without a blender. We extrapolated from previous published data that such flows in infants would generate fraction of inspired oxygen $\left(\mathrm{FiO}_{2}\right)$ values of 0.35 to 0.4 . The baseline characteristics of each participant were recorded in each participant's data collection record, which included heart rate, oxygen saturation, respiratory rate and M-TAL score.

Participants in both allocated groups were then reviewed 60 to 90 minutes later, and their HRs, RRs, saturations and work of breathing, re-assessed and recorded. Therapy was terminated if any of the following conditions were met: impending respiratory failure (indicated by fatigue, apnoea, $\mathrm{PaCO}_{2}>55 \mathrm{mmHg}$ with $\mathrm{pH}<7.25$ ), saturations $<90 \%$ despite $\mathrm{FiO}_{2} 0.6$, or altered mental state. Participants who met the criteria for termination of treatment were intubated and mechanically ventilated. There was no crossover to high-flow from standard therapy.

All other aspects of ongoing care for both groups were left up to the discretion of the treating physicians. Nursing staff recorded vitals on high care charts, and suctioned nostrils as clinically indicated. All feeding tubes were placed nasally, if required, and the decision to start feeds was left up to the discretion of the treating physicians.

Participants in both groups were then reviewed at 6- to 12-hourly intervals during the course of their stay in the high-care area. At each review by a clinician, the HR, RR, oxygen saturation and M-TAL were measured and recorded on the patient record sheet. Additionally, at each patient review, evidence of failure of therapy and the need for escalation of therapy was assessed. 
On initiation of this research study, HFHO therapy was not standard therapy at $\mathrm{CHBAH}$, nor was it available in the paediatric wards or highcare area of the paediatric admission ward, although it was available to infants admitted to the PICU. At the end of the first bronchiolitis season, and our first group of participant enrollments, the high-care unit obtained their own equipment and consumables to administer HFHO therapy. This development followed the publication of the results of a large RCT of highflow use in infants with moderate-to-severe bronchiolitis, which showed the benefit of HFHO therapy over standard therapy. ${ }^{[10]}$ This study enrolled 1400 infants under 1 year of age with moderate to severe bronchiolitis (oxygen saturations $<92 \%$ ) who were randomised to either HFHO therapy at $2 \mathrm{~L} / \mathrm{kg} / \mathrm{min}$ or low-flow oxygen via nasal cannula, which was almost identical to our study, albeit in a better resourced environment with greater access to intensive care facilities. At this juncture, the investigators felt it was unethical to continue to randomise patients to low-flow nasal cannula as 'standard therapy' in these patients with moderate-to-severe respiratory distress, when HFHO was now available to them as standard care. Subsequently the study was terminated early after enrolling 30 patients.

\section{Results}

Data were analysed for distribution and appropriate non-parametric statistical analysis performed. We used median and interquartile range (IQR) for continuous data and percentages for dichotomous data. The Mann-Whitney U-test was used to compare continuous data and the $\chi^{2}$ test was used for binary data. The Wilcoxon matched-pairs test was to compare changes within groups. The $5 \%$ significance level was used. Statistica version 13.3 (TIBCO Software Inc., USA) was used for the analysis.

Study enrolment took place between August 2017 and August 2018. A total of 67 infants were screened, of whom 30 were enrolled. Of those who were not enrolled, one had no consent, 13 had exclusion criteria, and 23 were too well (had M-TAL scores $<6$ and oxygen saturations $>92 \%$ on room air, despite being admitted to the high-care area.) Two of those who were initially enrolled were later excluded due to previous ventilation during current hospital admission, and the other was confirmed to have a complex congenital heart lesion after enrolment (Fig. 1).

Fifteen patients were randomised to the high-flow nasal cannula group (HFNC) and 13 patients to the control group. Baseline characteristics

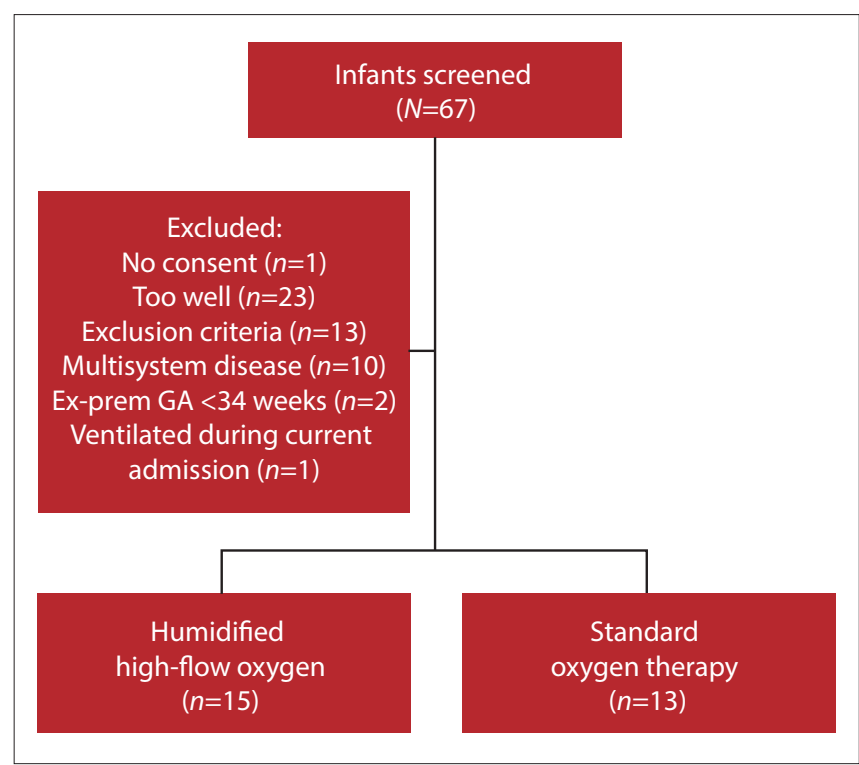

Fig. 1. Patient screening and enrolment. ( $G A=$ gestational age. $)$ are given in Table 1. Arterial blood gas data were not available for all participants. A gradient was calculated using estimated $\mathrm{FiO}_{2}$ as previously described.

\section{Respiratory changes}

The M-TAL score, which was a composite of the RR, cyanosis, wheezing and accessory muscle use improved significantly over the first 24 hours only in the HFNC group. (Table 2)

There were trends to a decrease in the number of days on oxygen therapy and intubation rates in the HFNC group compared with the control group. None of these reached statistical significance. There was no difference in length of hospital stay between the two groups (Table 3).

\section{Table 1. Baseline characteristics}

\begin{tabular}{|c|c|c|c|c|}
\hline & $\begin{array}{l}\text { HFHO, } \\
\text { median }(\mathrm{IQR})^{\star}\end{array}$ & $N$ & $\begin{array}{l}\text { Control, } \\
\text { median }(\mathrm{IQR})^{\star}\end{array}$ & $N$ \\
\hline Age (months) & $4(2-11)$ & 15 & $5(3-9)$ & 13 \\
\hline Weight (kg) & $8(5.6-9.9)$ & 15 & $6(5.6-8.1)$ & 13 \\
\hline Days ill prehospital & $2(1-4)$ & 15 & $2(1-2)$ & 13 \\
\hline RR & $63(62-68)$ & 15 & $64(60-82)$ & 13 \\
\hline M-TAL & $7(6-9)$ & 15 & $7(6-8)$ & 13 \\
\hline $\mathrm{FiO}_{2}$ & $0.4(0.4-0.4)$ & 15 & $0.4(0.4-0.4)$ & 13 \\
\hline PF ratio & $235(167-397)$ & 9 & $185(148-265)$ & 11 \\
\hline $\mathrm{A}-\mathrm{aDO}_{2}$ & $87(32-127)$ & 9 & $112(78-188)$ & 11 \\
\hline $\mathrm{pH}$ & $7.42(7.39-7.45)$ & 9 & $7.33(7.30-7.43)$ & 11 \\
\hline $\mathrm{PaO}_{2}, \mathrm{mmHg}$ & $111(87-159)$ & 9 & $89(73-128)$ & 11 \\
\hline $\mathrm{PaCO}_{2}, \mathrm{mmHg}$ & $38(34-39)$ & 9 & $47(38-52)$ & 11 \\
\hline Viral PCR done, $n$ & 7 & 15 & 7 & 13 \\
\hline Viral PCR positive, $n$ & 5 & 15 & 6 & 13 \\
\hline Adenovirus & 2 & & 1 & \\
\hline RSV & 2 & & 4 & \\
\hline Coronavirus & 1 & & 0 & \\
\hline Rhinovirus & 0 & & 1 & \\
\hline \multicolumn{5}{|c|}{$\begin{array}{l}\mathrm{HFHO}=\text { high-flow humidified oxygen } \mathrm{IQR}=\text { interquartile range; } \mathrm{RR}=\text { respiratory } \\
\text { rate; } \mathrm{M}-\mathrm{TAL}=\text { modified } \mathrm{TAL} \text { score; } \mathrm{FiO}=\text { fraction of inspired oxygen; } \\
\mathrm{PF}=\mathrm{PaO}_{2} / \mathrm{FiO}_{2} ; \mathrm{A}-\mathrm{aDO}_{2}=\text { alveolar-arterial oxygen gradient; } \mathrm{PaO}=\text { arterial partial } \\
\text { pressure of oxygen; } \mathrm{PaCO}_{2}=\text { arterial partial pressure of carbon dioxide, } \\
\mathrm{PCR}=\text { polymerase chain reaction; } \mathrm{RSV}=\text { respiratory syncytial virus. } \\
\text { *Unless otherwise specified. }\end{array}$} \\
\hline
\end{tabular}

Table 2. Respiratory changes (M-TAL score) from baseline to 24 hours

\begin{tabular}{lllll}
\hline & $\begin{array}{l}\text { M-TAL score } \\
\text { at baseline }\end{array}$ & $\begin{array}{l}\text { M-TAL score } \\
\text { at 24 hours }\end{array}$ & $\boldsymbol{N}$ & p-value \\
\hline HFHO, median (IQR) & $7(3)$ & $3(4)$ & 15 & $0.04^{*}$ \\
Control, median (IQR) & $7(2)$ & $5(10)$ & 12 & 0.69 \\
& & & & \\
& \\
M-TAL = modified TAL; HFHO = high-flow humidified oxygen; IQR = interquartile \\
range. \\
*Significant $p$-value.
\end{tabular}

Table 3. Patient outcomes

\begin{tabular}{llllll}
\hline $\begin{array}{l}\text { Respiratory } \\
\text { support }\end{array}$ & $\begin{array}{l}\text { HFHO, } \\
\text { median (IQR)* }\end{array}$ & $\boldsymbol{N}$ & $\begin{array}{l}\text { Control, } \\
\text { median (IQR) }\end{array}$ & $\boldsymbol{N}$ & p-value \\
\hline $\begin{array}{l}\text { Days on oxygen } \\
\text { LOS of survivors } \\
\text { (days) }\end{array}$ & $8.5(3.25-6.75)$ & 15 & $6(7)$ & 13 & 0.7 \\
$\begin{array}{l}\text { Intubation rate, } \\
n(\%)\end{array}$ & $3(20)$ & 15 & $8(9)$ & 11 & 0.44 \\
$\begin{array}{l}\text { HFHO = High-flow humidified oxygen; IQR = interquartile range; LOS = length of stay. } \\
\text { *Unless otherwise specified. }\end{array}$ & 15 & $6(46)$ & 13 & 0.139 \\
\end{tabular}




\section{Cardiovascular changes}

HR decreased significantly within 60 to 90 minutes in the HFNC group compared with the control group. This decrease persisted as a trend at 4 to 6 hours, although it did not meet statistical significance (Table 4).

\section{Discussion}

This study showed that the use of humidified high-flow nasal cannula oxygen in infants with moderate to severe respiratory distress, resulted in a reduction in respiratory distress in these infants, as indicated by a reduction in their M-TAL scores. The M-TAL score is a validated tool to assess the severity of respiratory distress in bronchiolitis, and is widely used. ${ }^{[11]}$ In addition, it demonstrates good inter-observer reliability and consistency. ${ }^{[11]}$

Our findings of improved work of breathing, are in keeping with other studies in infants (both prospective and retrospective) with bronchiolitis which have shown that HFHO results in a reduction in $\mathrm{RR}$, effort and improvement of breathing, and improvement in blood gas parameters. ${ }^{[12-16]}$

In a prospective observational study among 36 infants with moderatesevere bronchiolitis, there was a significant reduction in respiratory rate in those who received HFHO compared with those who received low-flow oxygen, ${ }^{[15]}$ while others demonstrated an improvement in COMFORT and RDS scores in infants with bronchiolitis who received HFHO therapy. ${ }^{[13]}$

In a retrospective observational cohort of infants with bronchiolitis admitted to an ICU over 2 seasons, the application of HFHO resulted in a decrease in respiratory rate after 1 hour on the therapy. ${ }^{[13]}$

The improvement in work of breathing which occurs with HFHO therapy is thought to occur for several reasons. ${ }^{[6]}$ Physiological studies have demonstrated that the application of $\mathrm{HFHO}$ at flows of $7 \mathrm{~L} / \mathrm{min}$ are able to produce a positive pharyngeal pressure, and a reduction in the oesophageal pressure swings which occur in spontaneously breathing patients. ${ }^{[6]}$ The beneficial effect of this is two-fold: it results in a reduction in work of breathing, by offloading work on the respiratory muscles, (a reduction in the area under the curve in the pressure-time curve for inspiratory effort, which is a validated marker of work of breathing), as well as by modifying breathing patterns by producing longer expiratory times and lower respiratory rates per minute. ${ }^{[6,17]}$

Additionally, our study demonstrated that the use of HFHO therapy in infants with bronchiolitis and moderate-severe respiratory distress also resulted in cardiovascular improvement, by reducing the HR. This improvement occurred within the first 24 hours of the therapy being applied.

A retrospective review of 167 infants with viral bronchiolitis treated with HFHO in their ICU over a 5-year period, in infants with viral bronchiolitis, demonstrated a reduction in the mean RR and HR by

Table 4. HR and changes in HR in the HFHO and control groups

\begin{tabular}{llllll}
\hline & $\begin{array}{l}\text { HFHO, } \\
\text { median (IQR) }\end{array}$ & $\boldsymbol{N}$ & $\begin{array}{l}\text { Control, } \\
\text { median }(I Q R)\end{array}$ & $\boldsymbol{N}$ & $\boldsymbol{p}$-value \\
\hline HR 0 (/min) & $164(17)$ & 15 & $168(12)$ & 13 & 0.41 \\
HR 1 (/min) & $150(23)$ & 15 & $167(22)$ & 13 & $0.005^{*}$ \\
HR 2 (/min) & $152(19)$ & 15 & $160(19)$ & 9 & 0.34 \\
DHR 0 - 1 & $-10(22)$ & 15 & $0(9)$ & 13 & $0.029^{*}$ \\
DHR 0 - 2 & $-16(33)$ & 15 & $-10(15)$ & 9 & 0.89
\end{tabular}

$\mathrm{HR}=$ heart rate; $\mathrm{HFHO}=$ high-flow humidified oxygen; $\mathrm{IQR}=$ interquartile range; $\mathrm{HR} 0=\mathrm{HR}$ at baseline, HR $1=\mathrm{HR}$ at $60-90 \mathrm{~min}, \mathrm{HR} 2=\mathrm{HR}$ at $4-6$ hours, DHR = change in HR.

${ }^{\star}$ Significant $p$-value. more than $20 \%$ from baseline, which is in keeping with the findings of our study. ${ }^{[18]}$

Mayfield et al. ${ }^{[7]}$ also demonstrated improvements in both respiratory and heart rates among infants with bronchiolitis who were given $\mathrm{HFHO}$ at $2 \mathrm{~L} / \mathrm{kg} / \mathrm{min}$. They proposed that failure of heart rate and respiratory rate to improve after 60 minutes of treatment identified patients as non-responders to therapy (for both high-flow and control groups).

We also demonstrated a trend to lower intubation rates in the group of patients treated with HFHO compared with lowflow oxygen. Two other retrospective studies among infants with bronchiolitis have demonstrated similar findings. Following the introduction of HFHO in one PICU, the intubation rates among infants with viral bronchiolitis reduced from $37 \%$ to $7 \%$, despite an increase in the numbers of infants who were admitted to the unit with bronchiolitis. ${ }^{[19]}$ In another unit, the introduction of HFHO resulted in a reduction of intubation rates from $23 \%$ to $9 \%$ from one bronchiolitis season to the next. ${ }^{[13]}$

The cost of the humidified high-flow consumables is higher than a standard low-flow nasal cannula device. However, in one institution, the introduction of a hospital-wide high-flow nasal cannula protocol, which included the implementation of HFHO use in the general paediatric wards, they were able to reduce length of hospital stay as well as median total hospital charges for these patients. ${ }^{[20]}$ We did not demonstrate a reduction in length of hospital stay in our study, which was most likely due to the early termination of the study and reduced number of patients.

The infants in our study had been ill for an average of 2 days before presenting to the hospital, and had a mean Modified TAL score of 7 , which is interpreted as moderate respiratory distress. The HFHO was administered outside the intensive care unit, in the high-care area of the paediatric admission ward.

Limited resources in our hospital mean that the intensive care unit usually only accepts patients who require mechanical ventilation, and even then we are only able to accommodate $63.3 \%$ of requests for intensive care beds. ${ }^{[9]}$

The high-care area where our study was conducted accommodates patients who are not yet ventilated and have moderate to severe bronchiolitis. The trend to lower intubation rates and cardiovascular stabilising effect may have a sparing effect on intensive care resource utilisation. HFHO therapy therefore shows promise, especially in the context of limited healthcare resources, for the management of infants with moderate-severe viral bronchiolitis.

The small sample size was a limitation of our study. Additionally, convenience sampling, necessitated by lack of full-time research staff, was a limitation, because potential participants who presented after hours or on weekends were not included.

Other aspects of care, such as feeds and fluids, as well as whether viral specimens for PCR were sent, were left up to the discretion of the treating doctor. Therefore, we do not have complete information about viral aetiology and the amounts of intravenous fluids received by these patients, which may have been important in predicting therapy failure.

\section{Conclusion}

In infants with viral bronchiolitis and moderate-severe respiratory distress, HFHO is a beneficial therapy which can be safely used outside the ICU. We demonstrated that it produces a rapid improvement in the heart rate, as well as an objective improvement in respiratory distress (M-TAL score) and a trend to a lower intubation rate when compared 
with standard oxygen delivery systems. It may potentially reduce the the need for intubation and ventilation and admission to ICU. Further research into whether other groups of children with respiratory pathology other than viral bronchiolitis will benefit from HFHO in lowresource settings is needed.

Declaration. We confirm that the manuscript has been read and approved by all named authors.

Acknowledgements. Dr G Okudo, Prof. Z Dangor and the Department of Paediatrics, as well as Prof. LRM Mathivha (Department of Critical Care, Chris Hani Baragwanath Academic Hospital) for their support of this research study.

Author contributions. SM: conceptualisation, data collection, data interpretation, drafting article, critical revision of manuscript and approval of final version. SO: data analysis and interpretation, critical revision of manuscript and approval of final version. AK, AS, EB, LD: data collection, critical revision of manuscript, approval of final version.

Funding. None. The consumables for the high-flow circuits were donated by Fisher and Paykel Healthcare, NZ.

Conflicts of interest. None.

1. Mayfield S, Jauncey-Cooke J, Bogossian F. A case series of paediatric high flow nasal cannula therapy. Aust Crit Care 2013 Nov;26(4):189-192. https://doi.org/10.1016/j.aucc.2013.05.004

2. Dysart K, Miller TL, Wolfson MR, Shaffer TH. Research in high flow therapy: Mechanisms of action. Respir Med 2009;103(10):1400-1405. https://doi.org/10.1016/j.rmed.2009.04.007

3. Milési C, Boubal M, Jacquot A, et al. High-flow nasal cannula: Recommendations for daily practice in pediatrics. Ann Intensive Care 2014;4(1):29. https://doi.org/10.1186\%2Fs13613-014-0029-5

4. Hasani A, Chapman TH, McCool D, Smith RE, Dilworth JP, Agnew JE. Domiciliary humidification improves lung mucociliary clearance in patients with bronchiectasis. Chron Respir Dis 2008;5(2):81-86. https://doi.org/10.1177/1479972307087190

5. Fontanari P, Burnet $\mathrm{H}$, Zattara-Hartmann MC, Jammes Y. Changes in airway resistance 1996;81(4):1739-1743. https://doi.org/10.1152/jappl.1996.81.4.1739
6. Milési C, Baleine J, Matecki S, et al. Is treatment with a high flow nasal cannula effective in acute viral bronchiolitis? A physiologic study. Intensive Care Med 2013;39(6):1088-1094. https://doi. org/10.1007/s00134-013-2879-y

7. Mayfield S, Bogossian F, O’Malley L, Schibler A. High-flow nasal cannula oxygen therapy for infants with bronchiolitis: Pilot study. J Paediatr Child Health 2014;50(5):373-378. https://doi. org/10.1111/jpc.12509

8. Kepreotes E, Whitehead B, Attia J, et al. High-flow warm humidified oxygen versus standard low-flow nasal cannula oxygen for moderate bronchiolitis (HFWHO RCT): An open, phase 4 , randomised controlled trial. Lancet 2017;389(10072):930-939. https://doi.org/10.1016/s01406736(17)30061-2

9. Cawood, SK, Naidoo S, Okudo G, et al. Outcomes of paediatric patients ventilated in a high care area outside an intensive care unit. S Afr Med J 2020;110(9):903-909.

10. Franklin D, Babl FE, Schlapbach LJ, et al. A randomised trial of high-flow oxygen therapy in infants with bronchiolitis. N Engl J Med 2018;378(12):1121-1131. https://doi.org/10.1056 nejmoal714855

11. McCallum GB, Morris PS, Wilson CC, et al. Severity scoring systems: Are they internally valid, reliable and predictive of oxygen use in children with acute bronchiolitis? Pediatr Pulmono 2013;48(8):797-803. https://doi.org/10.1002/ppul.22627

12. Bressan S, Balzani M, Krauss B, Pettenazzo A, Zanconato S, Baraldi E. High-flow nasal cannula oxygen for bronchiolitis in a pediatric ward: A pilot study. Eur J Pediatr 2013;172(12):1649-1656. https://doi.org/10.1007/s00431-013-2094-4

13. McKiernan C, Chua LC, Visintainer PF, Allen H. High flow nasal cannulae therapy in infants with bronchiolitis. J Pediatr 2010;156(4):634-638. https://doi.org/10.1016/j.jpeds.2009.10.039

14. Spentzas T, Minarik M, Patters AB, Vinson B, Stidham G. Children with respiratory distress treated with high-flow nasal cannula. J Intensive Care Med 2009;24(5):323-328. https://doi. org/10.1177/0885066609340622

15. Kallappa C, Hufton M, Millen G, Ninan TK. Use of high-flow nasal cannula oxygen (HFNCO) in infants with bronchiolitis on a paediatric ward: A 3-year experience. Arch Dis Child 2014;99(8):790-791. https://doi.org/10.1136/archdischild-2014-306637

16. Milani GP, Plebani AM, Arturi E, et al. Using a high-flow nasal cannula provided superior results to low-flow oxygen delivery in moderate to severe bronchiolitis. Acta Paediatr 2016;105(8):e368372. https://doi.org/10.1111/apa.13444

17. Cambonie G, Milési C, Jaber S, et al. Nasal continuous positive airway pressure decreases respiratory muscles overload in young infants with severe acute viral bronchiolitis. Intensive Care Med 2008;34(10):1865-1872. https://doi.org/10.1007/s00134-008-1201-x

18. Pham TMT, O'Malley L, Mayfield S, Martin S, Schibler A. The effect of high flow nasal cannula therapy on the work of breathing in infants with bronchiolitis. Pediatr Pulmonol 2015;50(7):713720. https://doi.org/10.1002/ppul.23060

19. Schibler A, Pham TMT, Dunster KR, et al. Reduced intubation rates for infants after introduction of high-flow nasal prong oxygen delivery. Intensive Care Med 2011;37(5):847-852. https://doi. org/10.1007/s00134-011-2177-5

20. Riese J, Fierce J, Riese A, Alverson BK. Effect of a hospital-wide high-flow nasal cannula protocol on clinical outcomes and resource utilisation of bronchiolitis patients admitted to the PICU. Hosp Pediatr 2015;5(12):613-618. https://doi.org/10.1542/hpeds.2014-0220

Accepted 20 May 2020. 